\title{
Filter Augmented JPEG Algorithms: A Critical Performance Study for Improving Bandwidth
}

\author{
Ch. Ramesh \\ Professor, Dept .of CSE \\ AITAM, Tekkali, A.P, India
}

\author{
N.B. Venkateswarlu, PhD. \\ Professor, Dept .of CSE \\ AITAM, Tekkali, A.P, India
}

\author{
J.V.R. Murthy, PhD. \\ Professor, Dept .of CSE \\ College of Engineering, \\ JNTUK, A.P, India
}

\begin{abstract}
In the recent years, use of Digital Image Communication has increased exponentially in the day to day activities. Joint Photographic Experts Group (JPEG) is the most widely used still image compression standard for bandwidth conservation. In this paper, it is proposed and critically studied a new set of JPEG Compression algorithms by combining Mean filtering, Median filtering, and Outlier detection algorithms and conventional JPEG DCT algorithm in a staged manner. This outlier based JPEG algorithm is giving exceptionally compression compared to conventional JPEG algorithm. Experiments are carried out with many standard still images. Algorithms developed in this paper, identified to be giving almost same Peak Signal-to-Noise Ratio (PSNR) as that of standard JPEG algorithm.
\end{abstract}

Key words:-Mean, Median, Outlier, DCT, IDCT, PSNR, JPEG

\section{INTRODUCTION}

In the recent years, data intensive multimedia-based web applications have increased by many folds. Many remote applications such as remote monitoring, surveillance, automatic navigation systems often involves communication of captured images or videos for further processing. In these applications, it is inevitable to conserve the available bandwidth in order to reduce consumer side bills. In order to reduce the bills (or bandwidth consumption), communicating compressed images is a classical solution in communications and allied fields. In the literature, various algorithms were proposed for the image compression, and even many of them are available in embedded HW also. JPEG compression is the standard for image compression for still images. Its variants are under the name hood of MPEG (Motion Picture Experts Group) is widely followed standard for video encoding.

Image based recognition systems that are used in production industries such as IC manufacturing, fruit processing systems, automatic welding, etc., will be using original image. However, there are some recent applications such as remote monitoring, surveillance, remote surgery, etc., may involve communication of captured images to a server for processing, recognition and control. For example, [7] used compressed face images in JPEG format for the development of their face recognition system that extracts edge based features from the JPEG images and uses with a neural network. Certainly, performance of recognition system based on original images will be giving better results than compressed images as compressed images loses some details which are otherwise useful for recognition. It is reported elsewhere [12] JPEG compression induces some artifacts such as noise around edges, blurring, a smeared appearance, color distortion, and/or checkerboard-like blocking in busy regions. However, it consumes very little bandwidth. Thus, scientific community may be interested in studying about recognition system performance when it is designed to use compressed images rather than original images. Authors [13] reports that JPEG images cannot be used for character recognition as JPEG distorts the sharp edges.

Herewith the authors propose a new set of image compression algorithms which uses Mean filtering, Median filtering, and Outlier detection concepts with conventional DCT algorithm in a staged manner that shows better edge images compared to conventional JPEG images. The average peak signal to noise ratio (PSNR) [1] of our algorithms is compared with original JPEG method.

The paper is organized as follows. In section 2, a brief overview of the JPEG standard is provided. The proposed Mean, Median \& Outlier based JPEG compression system is described in section 3. Experimental results are presented in section 4. Finally, conclusions are reported in section 5.

\section{BRIEF OVERVIEW OF JPEG ENCODING / DECODING SYSTEM}

JPEG is a well known standardized image compression technique. JPEG loses information so the decompressed picture is not the same as the original one. The main reason for use of JPEG is to reduce the size of image files. Reducing image files is an important procedure for transmitting files across networks or archiving libraries. Usually JPEG can remove the less important data before the compression; hence JPEG is able to compress images meaningfully, which produces a huge difference in the transmission time and the disk space. Fig 1 shows the basic Architecture of JPEG compression system. Here is a brief overview of the JPEG compression system. [2]

The image is first subdivided into pixel blocks of size $8 \mathrm{X} 8$, which are processed left to right and top to bottom. As each $8 \mathrm{X} 8$ block or sub image is encountered, its 64 pixels are level shifted by subtracting the quantity $\mathrm{L} / 2$, where $\mathrm{L}$ is the Gray level resolution of the image. The 2-D Forward Discrete Cosine Transform (FDCT) (Eq 1) [5] of the block is then computed, quantized using 64 corresponding step size values from the quantization table in Fig.2[3]. After quantization the DCT coefficients are rearranged in a zigzag sequence order as shown in the Fig.3. [3] 
Since the one-dimensional reordered array generated under the zigzag pattern of Fig. 3 is qualitatively arranged according to increasing spatial frequency, the JPEG coding procedure is designed to take the advantage of the long runs of zeros that normally result from the reordering. In particular, the nonzero AC coefficients (the term AC denotes all transform coefficients with the exception of the zero ${ }^{\text {th }}$ or DC coefficient) are coded using a variable-length code that defines the coefficient's value and number of preceding zeros. The DC coefficient is difference coded relative to the DC coefficient of the previous sub image.

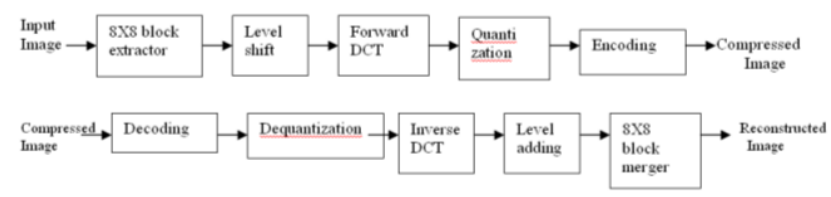

Fig. 1 Basic Architecture of JPEG Compression

The 2-D DCT is

$C(u, v)=\alpha(u) \alpha(v) \sum_{x=0}^{N-1} \sum_{y=0}^{N-1} f(x, y) \cos \left[\frac{(2 x+1) u \pi}{2 N}\right] \cos \left[\frac{(2 y+1) v \pi}{2 N}\right]$

for $\mathrm{u}, \mathrm{v}=0,1,2, \ldots, \mathrm{N}-1$

$\alpha(u)=\left\{\begin{array}{lll}\sqrt{1 / N} & \text { for } & u=0 \\ \sqrt{2 / N} & \text { for } & u>0\end{array}\right.$
$\alpha(v)=\left\{\begin{array}{lll}\sqrt{1 / N} & \text { for } & v=0 \\ \sqrt{2 / N} & \text { for } & v>0\end{array}\right.$

\begin{tabular}{|l|l|l|l|l|l|l|l|}
\hline 16 & 11 & 10 & 16 & 24 & 40 & 51 & 61 \\
\hline 12 & 12 & 14 & 19 & 26 & 58 & 60 & 55 \\
\hline 14 & 13 & 16 & 24 & 40 & 57 & 69 & 56 \\
\hline 14 & 17 & 22 & 29 & 51 & 87 & 80 & 62 \\
\hline 18 & 22 & 37 & 56 & 68 & 109 & 103 & 77 \\
\hline 24 & 35 & 55 & 64 & 81 & 104 & 113 & 92 \\
\hline 49 & 64 & 78 & 87 & 103 & 121 & 120 & 101 \\
\hline 72 & 92 & 95 & 98 & 112 & 100 & 103 & 99 \\
\hline
\end{tabular}

Fig.2 Quantization Matrix [3]
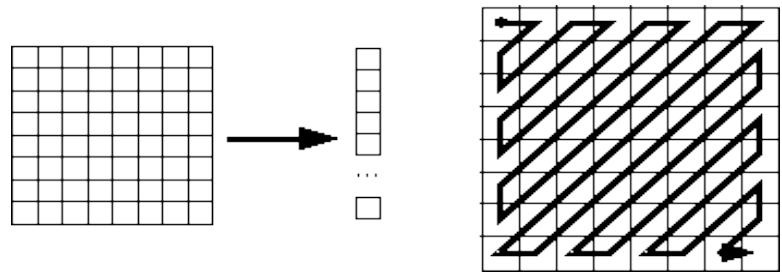

Fig.3 Zig Zag Sequence [3]

The decompression process performs an inverse procedure. It decodes the Huffman codes. Then, it makes the inversion of the Quantization step. In this stage, the decoder raises the small numbers by multiplying them by the quantization coefficients. The results are not accurate, but they are close to the original numbers of the DCT coefficients. An Inverse Discrete Cosine Transform (IDCT) (Eq.4) [6] is performed on the data received from the previous step. Finally add L/2 to each sub image. Place the sub images in their correct positions.

$\hat{f}(x, y)=\sum_{u=0}^{N-1} \sum_{v=0}^{N-1} \alpha(u) \alpha(v) C(u, v) \cos \left[\frac{(2 x+1) u \pi}{2 N}\right] \cos \left[\frac{(2 y+1) v \pi}{2 N}\right]$

The error between the original image and reconstructed image is calculated in terms of Peak signal to noise ratio $(\mathrm{PSNR})=10 \log _{10}\left(\mathrm{~L}^{2} / \mathrm{MSE}\right)$

$$
\begin{aligned}
& M S E=\frac{\sum_{x=0}^{m-1} \sum_{y=0}^{n-1}[\hat{f}(x, y)-f(x, y)]^{2}}{m X n} \\
& \text { MSE - Mean Squared Error } \\
& \hat{f}(x, y) \text { - Reconstructed Image } \\
& \mathrm{f}(\mathrm{x}, \mathrm{y})-\text { Original Image } \\
& \mathrm{m} \text { x } \mathrm{n}-\text { Size of the Image }
\end{aligned}
$$

\section{NEW MEAN, MEDIAN \& OUTLIER BASED JPEG ALGORITHMS}

Mean filtering [8] is a simple, intuitive and easy to implement method of image smoothing i.e. reducing the amount of variation between one pixel and the next or surrounding pixels. It is often used to reduce noise in image. The idea of mean filtering is simply to replace each pixel in an image with the mean value of its neighbors including itself. This has the effect of eliminating pixel values which are unrepresentative of their surroundings. Usually, $3 \times 3$ neighborhoods of pixels are considered while calculating mean filtered value of any pixel.

Median filter [9] is normally used to reduce noise in an image like the mean filter. However, it often does a better job than the mean filter in preserving useful detail in the image. Like the mean filter, the median filter considers each pixel in the image in turn and looks at its neighbors to decide whether or not its representative of its surroundings. Instead of simply replacing the pixel value with the mean of neighboring pixel values, it replaces it with the median of those values.

An outlier [11] is an observation that is numerically distant from the rest of the data. In an image, a pixel value is very different from its surrounding pixels, it can be called as outlier. Certainly, replacing its value with mean filtered or median filtered or DCT based with induce noise into our image. Thus, the authors propose to retain its value as it is such that noise will become less and more over subsequent edge detection results will be attractive for image recognition systems.

From basic statistics, authors know that populations sample values with some confidence level can be given as mean \pm $\mathrm{C}^{*} \sigma$, where $\mathrm{C}$ is weighing factor (critical value) and $\sigma$ is standard deviation of the population. Table-1 shows the commonly used Confidence Levels and Corresponding 
Critical Values [10]. In the outlier based algorithms, take this simple confidence limits of normal distribution in deciding whether a pixel is outlier or not. If the pixel is observed to be outlier with the given confidence level, one may retain else may take its mean filtered or median filtered value.

Table 1: Confidence Levels Vs Critical values

\begin{tabular}{|l|c|c|c|c|c|c|c|}
\hline Confidence Level & $80 \%$ & $90 \%$ & $95 \%$ & $98 \%$ & $99 \%$ & $99.80 \%$ & $99.90 \%$ \\
\hline Critical Values & 1.28 & 1.645 & 1.96 & 2.33 & 2.58 & 3.08 & 3.27 \\
\hline
\end{tabular}

In the following, the authors listed the basic Mean, Median and outlier algorithms

\subsection{MeanDCT Algorithm}

1. Apply mean filtering to the original image using $3 \times 3$ window

2. Apply DCT on the mean filtered images

\subsection{MedianDCT Algorithm.}

1. Apply median filtering to the original image using $3 \times 3$ window

2. Apply DCT on the mean filtered images

\subsection{Outlier MeanDCT Algorithm}

1. Apply mean filtering with a little variation to the given original image using $3 \times 3$ window. For each pixel, calculate average and standard deviation of its neighboring $3 \times 3$ pixels. If a pixels value is observed to be outlier (not in the range of Mean $\pm C^{*} \sigma$ ) then its filtered value is taken as itself else mean is taken as its filtered value.

2. Apply DCT on the mean filtered image.

\subsection{Outlier MedianDCT Algorithm}

1 Apply median filtering with a little variation to the given original image using $3 \times 3$ window. For each pixel, calculate average and standard deviation of its neighboring $3 \times 3$ pixels. If a pixels value is observed to be outlier (not in the range of Mean $\pm C^{*} \sigma$ ) then its filtered value is taken as itself else median is taken as its filtered value.

2 Apply DCT on the median filtered image.

\section{EXPERIMENTAL WORK}

In this study, the authors have used a number of images in tiff format from USC-SIPI image database "http://sipi.usc.edu/database" [4]. Experiments are carried out under MS Windows XP version 2002, SP3 edition. The experimental system is equipped with Intel core 2 Duo 2.60 $\mathrm{GHz}$ processor with $1 \mathrm{~GB}$ RAM. Programs are written in C language under Microsoft Visual Studio 2005 version 8.0. Table 2 illustrates conventional JPEG compression ratios and PSNR values of the selected images [4]
Table 2: Compression ratios and PSNR values of the selected images with conventional JPEG.

\begin{tabular}{|c|c|c|c|}
\hline \multirow[b]{2}{*}{ IM AGE } & \multirow[b]{2}{*}{ SIZE } & \multicolumn{2}{|c|}{ CONVENTIONAL ENCODING } \\
\hline & & Compression Ratio & PSNR \\
\hline Apc & $512 \times 512$ & 13.057 & 34.7217 \\
\hline Brickwall6 & $512 \times 512$ & 13.96 & 35.751 \\
\hline Car1 & $512 \times 512$ & 10.23 & 34.99 \\
\hline Grass3 & $512 \times 512$ & 13.14 & 33.839 \\
\hline Gravel & $512 \times 512$ & 9.3 & 34.771 \\
\hline Lena & $512 \times 512$ & 12.53 & 35.793 \\
\hline Peppers & $512 \times 512$ & 12.31 & 34.7604 \\
\hline Raffia & $512 \times 512$ & 13.14 & 33.839 \\
\hline Tank1 & $512 \times 512$ & 8.39 & 32.0536 \\
\hline Tank & $512 \times 512$ & 10.24 & 33.578 \\
\hline Truck & $512 \times 512$ & 11.12 & 37.428 \\
\hline Woolen & $512 \times 512$ & 6.21 & 29.088 \\
\hline Airplane2 & $1024 \times 1024$ & 15.66 & 34.4 \\
\hline Airport & $1024 \times 1024$ & 8.39 & 31.174 \\
\hline Bark2 & $1024 \times 1024$ & 6.85 & 31.7673 \\
\hline Glass & $1024 \times 1024$ & 12.9 & 37.248 \\
\hline Leather2 & $1024 \times 1024$ & 6.49 & 31.422 \\
\hline Man & $1024 \times 1024$ & 10.13 & 34.13 \\
\hline Metalgrates & $1024 \times 1024$ & 14.6 & 33.378 \\
\hline Plasticbubbles 2 & $1024 \times 1024$ & 7.97 & 32.289 \\
\hline Roof 2 & $1024 \times 1024$ & 11.12 & 33.368 \\
\hline Sand2 & $1024 \times 1024$ & 8.3 & 33.19 \\
\hline Sea & $1024 \times 1024$ & 18.54 & 38.236 \\
\hline Straw2 & $1024 \times 1024$ & 7.79 & 32.7811 \\
\hline Weave2 & $1024 \times 1024$ & 5.41 & 28.9874 \\
\hline Woodfence & $1024 \times 1024$ & 5.2 & 34.3204 \\
\hline
\end{tabular}

The authors have carried out extensive simulations with the selected images and proposed algorithms.Table 3 shows the Compression Benefit and PSNR values of MeanDCT algorithm Vs OutlierMeanDCT algorithm. With all the images it is found that MeanDCT and OutlierMeanDCT algorithms have better compression ratios as compared to conventional JPEG coding. The PSNR loss in MeanDCT and OutlierMeanDCT algorithms is negligible as compared to conventional JPEG coding. While comparing MeanDCT and the corresponding Outlier DCT, Compression Benefits are observed to be MeanDCT>OutlierMeanDCT (for $\mathrm{C}=1.28$ to 2.58). As the value of $\mathrm{C}$ increases in the Outlier, Compression Benefit increases. For $\mathrm{C}=3.08$ to 3.27 Compression Benefit in MeanDCT and OutlierMeanDCT is same. PSNR in MeanDCT<OutlierMeanDCT(for $\mathrm{C}=1.28$ to 2.58). As the value of $\mathrm{C}$ decreases in the Outlier, PSNR increases. For $\mathrm{C}=3.08$ to $3.27 \mathrm{PSNR}$ in MeanDCT and OutlierMeanDCT is same. 
Table 3: Compression Benefit and PSNR values of MeanDCT Vs Outlier Mean DCT

\begin{tabular}{|c|c|c|c|c|c|c|c|c|c|c|}
\hline \multirow{3}{*}{ Ima } & & \multirow{3}{*}{$\begin{array}{c}\text { Conven } \\
\text { tional }\end{array}$} & Mean & Outlier & Outlier & Outlier & Outlier & Outlier & Outlier & Outlier \\
\hline & & & DCT & Mean & Mean & Mean & Mean & Mean & Mean & Mean \\
\hline & & & & $\begin{array}{c}\text { DCT } \\
(\mathrm{C}=3.27)\end{array}$ & $\begin{array}{c}\text { DCT } \\
(\mathrm{C}=3.08)\end{array}$ & $\begin{array}{c}\text { DCT } \\
(\mathrm{C}=2.58)\end{array}$ & $\begin{array}{c}\text { DCT } \\
(\mathrm{C}=2.33)\end{array}$ & $\begin{array}{c}\text { DCT } \\
(\mathrm{C}=1.96)\end{array}$ & $\begin{array}{c}\text { DCT } \\
(\mathrm{C}=1.645)\end{array}$ & $\begin{array}{c}\text { DCT } \\
(\mathrm{C}=1.28)\end{array}$ \\
\hline & Compression Benefit in \% & & 29.49 & 29.49 & 29.49 & 28.74 & 27.2 & 23.63 & 18.91 & 11.88 \\
\hline & PSNR & 34.594 & 33.203 & 33.203 & 33.203 & 33.206 & 33.222 & 33.269 & 33.371 & 33.647 \\
\hline \multirow{2}{*}{ Brickwall6 } & Compression Benefit in \% & & 22.47 & 22.47 & 22.47 & 22.54 & 21.52 & 18.87 & 14.99 & 10.67 \\
\hline & \begin{tabular}{|l} 
PSNR \\
\end{tabular} & 35.751 & 34.597 & 34.597 & 34.597 & 34.599 & 34.605 & 34.626 & 34.723 & 34.961 \\
\hline \multirow{2}{*}{ Carl } & Compression Benefit in $\%$ & & 24.69 & 24.69 & 24.69 & 24.65 & 24.42 & 23.22 & 20.47 & 15.27 \\
\hline & $\begin{array}{l}\text { PSNR } \\
\end{array}$ & 34.99 & 33.542 & 33.542 & 33.542 & 34.543 & 33.548 & 33.596 & 33.73 & 34.024 \\
\hline \multirow{2}{*}{ Grass3 } & Compression Benefit in \% & & 36.5 & 36.5 & 36.5 & 36.22 & 34.85 & 29.47 & 22.33 & 12.82 \\
\hline & \begin{tabular}{|l} 
PSNR \\
\end{tabular} & 33.839 & 32.939 & 32.939 & 32.939 & 33.043 & 33.054 & 33.1 & 33.189 & 33.389 \\
\hline \multirow{2}{*}{ Gravel } & Compression Benefit in \% & & 18.92 & 18.92 & 18.92 & 18.9 & 18.76 & 18.05 & 16.53 & 12.78 \\
\hline & PSNR & 34.771 & 32.817 & 32.817 & 32.817 & 33.818 & 32.82 & 32.842 & 32.905 & 33.142 \\
\hline \multirow{2}{*}{ Lena } & Compression Benefit in \% & & 19.08 & 19.08 & 19.08 & 18.95 & 18.56 & 17.61 & 15.71 & 12.15 \\
\hline & PSNR & 35.793 & 32.937 & 32.937 & 32.937 & 33.936 & 32.936 & 32.952 & 33.009 & 33.347 \\
\hline \multirow{2}{*}{ Peppers } & Compression Benefit in $\%$ & & 19.2 & 19.2 & 19.2 & 19.02 & 18.51 & 16.8 & 14.4 & 11.06 \\
\hline & PSNR & 34.7604 & 32.105 & 32.105 & 32.105 & 32.106 & 32.197 & 32.126 & 32.176 & 32.307 \\
\hline \multirow{2}{*}{ Raffia } & Compression Benefit in \% & & 36.5 & 36.5 & 36.5 & 36.22 & 34.85 & 29.47 & 22.33 & 12.82 \\
\hline & \begin{tabular}{|l} 
PSNR \\
\end{tabular} & 32.839 & 33.039 & 33.039 & 33.039 & 33.043 & 33.954 & 33.1 & 33.189 & 33.389 \\
\hline \multirow{2}{*}{ Tank1 } & Compression Benefit in \% & & 28.55 & 28.55 & 28.55 & 28.45 & 28.12 & 26.13 & 22.05 & 15.06 \\
\hline & \begin{tabular}{|l|} 
PSNR \\
\end{tabular} & 32.0536 & 30.288 & 30.288 & 30.288 & 30.29 & 30.3 & 30.367 & 30.52 & 30.873 \\
\hline \multirow{2}{*}{ Tank } & Compression Benefit in \% & & 29.62 & 29.62 & 29.62 & 29.41 & 28.86 & 26.37 & 22.31 & 14.51 \\
\hline & $\begin{array}{ll}\text { PSNR } \\
\end{array}$ & 33.578 & 31.903 & 31.903 & 31.903 & 31.906 & 31.919 & 31.977 & 32.109 & 32.457 \\
\hline \multirow{2}{*}{ Truck } & Compression Ber & & 16.83 & 16.83 & 16.83 & 16.79 & 16.73 & 16.58 & 16.1 & 14.26 \\
\hline & PSNR & 37.428 & 36.886 & 36.886 & 36.886 & 35.888 & 35.892 & 35.902 & 35.938 & 36.081 \\
\hline \multirow{2}{*}{ Woolen } & Compression Benefit in \% & & 33.2 & 33.2 & 33.2 & 33.13 & 32.55 & 29.8 & 24.6 & 16.42 \\
\hline & & 29.088 & 26.715 & 26.715 & 26.715 & 26.716 & 26.729 & 26.819 & 27.026 & 27.473 \\
\hline \multirow{2}{*}{ Airplane2 } & Compression Benefit in \% & - & 35.38 & 35.38 & 35.38 & 34.73 & 32.86 & 27.62 & 20.73 & 11.77 \\
\hline & \begin{tabular}{|l|} 
PSNR \\
\end{tabular} & 34.4 & 33.5 & 33.5 & 33.5 & 33.502 & 33.508 & 33.524 & 33.585 & 33.79 \\
\hline \multirow{2}{*}{ Airport } & Compression Beneffit in $\%$ & & 30.67 & 67 & 30.67 & 30.53 & 29.98 & 27.63 & 23.38 & 16.13 \\
\hline & \begin{tabular}{|l} 
PSNR \\
\end{tabular} & 31.174 & 28.615 & 28.615 & 28.615 & 28.617 & 28.623 & 28.667 & 28.799 & 29.207 \\
\hline & Compression Benefit in \% & & 22.14 & 22.14 & 22.14 & 22.11 & 21.92 & 20.9 & 18.69 & 14.42 \\
\hline Bark2 & & 31.7773 & 29.625 & 29.625 & 29.625 & 29.625 & 29.634 & 29.693 & 29.841 & 30.185 \\
\hline & Compression Benefit in \% & & 34.73 & 34.73 & 34.73 & 34.69 & 34.38 & 33.78 & 32.84 & 30.07 \\
\hline & PSNR & 37.248 & 33.404 & 33.404 & 33.404 & 33.404 & 33.404 & 33.409 & 33.421 & 33.498 \\
\hline Tethen? & Compression Benefit in \% & & 21.46 & & & & & 20.38 & 18.57 & 15.66 \\
\hline & \begin{tabular}{|l|} 
PSNR \\
\end{tabular} & 31.422 & 28.473 & 28.473 & 28.473 & 28.473 & 28.478 & 28.523 & 28.651 & 29.016 \\
\hline Y & Compression Benefit in \% & & 22.74 & 22.74 & 22.74 & 22.52 & 22.07 & 20.69 & 18.16 & 13.62 \\
\hline Man & & 34.13 & & & & 158 & 1.17 & 31.214 & 31.359 & 31.771 \\
\hline Metal & Compression Benefit in \% & & 25.012 & 25.012 & 25.012 & 24.92 & 24.29 & 22.65 & 17.56 & 11.39 \\
\hline grates & $\begin{array}{l}\text { PSNR } \\
\end{array}$ & 33.378 & 32.302 & 32.302 & 32.302 & 32.302 & 32.311 & 32.765 & 32.424 & 32.644 \\
\hline & Compression Benefit in \% & & & & & & & & 18.53 & 13.57 \\
\hline & \begin{tabular}{|l} 
PSNR \\
\end{tabular} & 32.289 & 30.573 & 30.573 & 30.573 & 30.575 & 30.583 & 30.635 & 30.749 & 31.035 \\
\hline & Compression Benefit in $\%$ & & 29.65 & 29.65 & 29.65 & 29.46 & 28.53 & 24.92 & 20.36 & 14.35 \\
\hline & & 33.368 & 31.262 & 31.262 & 31.262 & 31.263 & 31.268 & 31.286 & 31.353 & 31.62 \\
\hline & Compression Benefit in \% & & 20.3 & 20.3 & 20.3 & 20.26 & 20 & 18.82 & 16.73 & 12.7 \\
\hline & PSNR & 33.19 & 31.616 & 31.616 & 31.616 & 31.617 & 31.626 & 31.673 & 31.783 & 32.04 \\
\hline & Compression Benefit in \% & & & & & 222.26 & 22.09 & 21.29 & 18.27 & 12.82 \\
\hline & \begin{tabular}{|c|} 
PSNR \\
\end{tabular} & 38.236 & 36.312 & 36.312 & 36.312 & 36.312 & 36.312 & 36.319 & 36.344 & 36.46 \\
\hline & Compression Benefit in $\%$ & & 18.27 & 18.27 & 18.27 & 18.25 & 18.09 & 17.31 & 15.65 & 12.43 \\
\hline & \begin{tabular}{|l|} 
PSNR \\
\end{tabular} & 32.7811 & 30.74 & 30.74 & 30.74 & 30.741 & 30.748 & 30.795 & 30.918 & 31.232 \\
\hline & Compression Benefit in \% & & 24.5 & 24.5 & 24.5 & 24.47 & 24.28 & 23.18 & 20.91 & 16.39 \\
\hline weave & PSNR & 28.9874 & 26.391 & 26.391 & 26.391 & 26.392 & 26.399 & 26.454 & 26.594 & 26.061 \\
\hline & Compression Beneffit in \% & & 23.98 & & & 233.86 & 23.2 & 20.61 & 16.69 & 10.84 \\
\hline & PSNR & 34.3204 & 32.761 & 32.761 & 32.761 & 32.762 & 32.77 & 32.805 & 32.891 & 33.161 \\
\hline
\end{tabular}

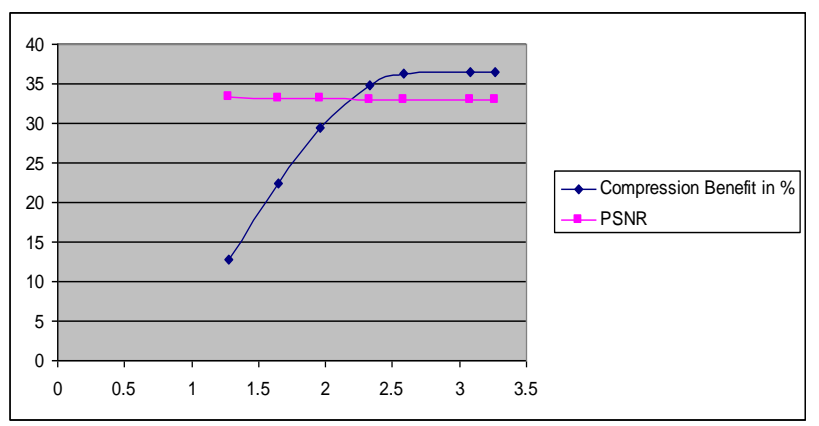

Fig. 4 : Critical Factor Vs Compression Benefit in \% \& Critical Factor Vs PSNR for Image "Gravel" with OutlierMeanDCT

Figure 4 shows As the $\mathrm{C}$ value increases, Compression Benefit increases and PSNR decreases. The variation in PSNR is very small as the $\mathrm{C}$ value increases. The PSNR values are very nearer to the PSNR values obtained by conventional JPEG coding.
Table 4 shows the Compression Benefit and PSNR values of MedianDCT algorithm Vs OutlierMedianDCT algorithm. With all the images it is found that MedianDCT and OutlierMedianDCT algorithms have better compression ratios as compared to conventional JPEG coding. The PSNR loss in MedianDCT and OutlierMedianDCT algorithms is negligible as compared to conventional JPEG coding. While comparing MedianDCT and the corresponding Outlier DCT, Compression Benefit in \%s are observed to be MedianDCT>OutlierMedianDCT(for $\mathrm{C}=1.28$ to 2.58 ). As the value of $\mathrm{C}$ increases in the Outlier, Compression Benefit increases. For $\mathrm{C}=3.08$ to 3.27 Compression Benefit in MedianDCT and OutlierMedianDCT is same. PSNR in MedianDCT<OutlierMedianDCT(for $\mathrm{C}=1.28$ to 2.58 ). As the value of $\mathrm{C}$ decreases in the Outlier, PSNR increases. For $\mathrm{C}=3.08$ to $3.27 \mathrm{PSNR}$ in MedianDCT and OutlierMedianDCT is Same.

Table 4 Compression Benefit and PSNR values of MedianDCT Vs OutlierMedianDCT

\begin{tabular}{|c|c|c|c|c|c|c|c|c|c|c|}
\hline \multirow{3}{*}{ Image } & & \multirow{3}{*}{$\begin{array}{c}\text { Conven } \\
\text { tional }\end{array}$} & \multirow{3}{*}{$\begin{array}{c}\text { MedianDC } \\
T \text { T }\end{array}$} & $\begin{array}{l}\text { Outlier } \\
\end{array}$ & Outlier & Outlier & Outlier & Outlier & Outlier & Outlier \\
\hline & & & & Median & Median & Median & Median & Median & Median & Median \\
\hline & & & & DCT & DCT & DCT & DCT & DCT & DCT & DCT \\
\hline \multirow{3}{*}{$\mathrm{Apc}$} & ompressisen & & & & $(\mathrm{C}=3.38)$ & $(\mathrm{C}=2.58)$ & $(C=2.33)$ & $(\mathrm{C}=1.96)$ & ${ }_{C}=1.645$ & $(\mathrm{C}=1.28)$ \\
\hline & enefersit in \% \% & & 27.58 & 27.58 & 27.58 & 26.9 & 25,57 & 22.14 & 17.58 & 10.65 \\
\hline & PSNR & 34.594 & 32.29 & 32.29 & 32.29 & 30.304 & 33.342 & 33.468 & 33.66 & 34.04 \\
\hline \multirow{2}{*}{ Brickwall6 } & $\begin{array}{l}\text { Compression } \\
\text { Benefitin }\end{array}$ & . & 17.34 & 17.34 & 17.34 & 16.79 & 16.37 & 14.4 & 11.41 & 7.22 \\
\hline & PSNR & 35.751 & 35.043 & 35.043 & 35.043 & 35.044 & 35.058 & 35.105 & 35.206 & 35.405 \\
\hline \multirow{2}{*}{ Carl } & $\begin{array}{l}\text { Compression } \\
\text { Benfetining }\end{array}$ & . & 20.5 & 20.5 & 20.5 & 20.44 & 20.27 & 19.12 & 16.4 & 11.08 \\
\hline & PSNR & 34.99 & 33.756 & 33.756 & 33.756 & 33.758 & 33.766 & 33.837 & 33.66 & 34.32 \\
\hline \multirow{2}{*}{ Grass 3} & Compression & . & 32.59 & 32.59 & 32.59 & 32.38 & 31.24 & 27.09 & 20.48 & 11.42 \\
\hline & 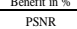 & 33.839 & 33.022 & 33.022 & 33.022 & 33.037 & 33.052 & 33.13 & 33.269 & 33.5 \\
\hline \multirow{2}{*}{ Gravel } & $\begin{array}{l}\text { Compression } \\
\text { Benefit in } \%\end{array}$ & . & 14.48 & 14.48 & 14.48 & 14.48 & 14.36 & 13.68 & 12.21 & 8.67 \\
\hline & PSNR & 34.771 & 33.022 & 33.022 & 33.022 & 33.421 & 33.423 & 33.468 & 33.546 & 33.79 \\
\hline \multirow{2}{*}{ Lena } & Compression & . & 13.21 & 13.21 & 13.21 & 13.15 & 12.89 & 12 & 10.45 & 7.26 \\
\hline & PSNR & 35.793 & 34.034 & 34.034 & 34.034 & 34.034 & 34.042 & 34.082 & 34.197 & 34.876 \\
\hline \multirow{2}{*}{ Peppers } & Compression & . & 12.82 & 12.82 & 12.82 & 12.66 & 12.25 & 10.71 & 8.61 & 5.4 \\
\hline & PSNR & 34.7604 & 33.691 & 33.691 & 33.691 & 33.694 & 33.71 & 33.753 & 33.862 & 34.665 \\
\hline \multirow{2}{*}{ Raffia } & Compression & . & 32.98 & 32.98 & 32.98 & 32.38 & 31.24 & 27.09 & 20.48 & 11.42 \\
\hline & PSNR & 32.839 & 33.122 & 33.122 & 33.122 & 33.027 & 33.052 & 33.13 & 32.269 & 33.5 \\
\hline \multirow{2}{*}{ Tank1 } & Compression & . & 24.4 & 24.4 & 24.4 & 24,29 & 23.91 & 22.05 & 18.22 & 11.67 \\
\hline & PSNR & 32.0536 & 30.405 & 30.405 & 30.405 & 30.41 & 30.428 & 30.522 & 30.73 & 31.162 \\
\hline \multirow{2}{*}{ Tank } & Compression & . & 25.78 & 25.78 & 25.78 & 25.64 & 25.12 & 22.8 & 18.78 & 11.47 \\
\hline & $\begin{array}{l}\text { PSNR } \\
\end{array}$ & 33.578 & 31.98 & 31.98 & 31.98 & 31.986 & 32.003 & 32.096 & 32.291 & 32.78 \\
\hline \multirow{2}{*}{ Truck } & $\begin{array}{l}\text { Compression } \\
\text { Paftion }\end{array}$ & . & 10.15 & 10.15 & 10.15 & 10.13 & 10.12 & 9.96 & 9.44 & 7.69 \\
\hline & $\begin{array}{l}\text { PSNR } \\
\end{array}$ & 37.428 & 36.597 & 36.597 & 36.597 & 36.6 & 36.602 & 36.611 & 36.655 & 36.808 \\
\hline \multirow{2}{*}{ Woolen } & Compression & & 28.96 & 28.96 & 28.96 & 28.91 & 28.37 & 25.93 & 21.25 & 13.44 \\
\hline & $\begin{array}{l}\text { PSNR } \\
\end{array}$ & 29.088 & 26.86 & 26.86 & 26.86 & 26.863 & 26.885 & 27.017 & 27.306 & 27.859 \\
\hline \multirow{2}{*}{ Airplane2 } & $\begin{array}{l}\text { Compression } \\
\text { Benefitin }\end{array}$ & & 32.24 & 32.24 & 32.24 & 31.82 & 30.27 & 25.68 & 19.64 & 11.11 \\
\hline & PSNR & 34.4 & 33.386 & 33.386 & 33.386 & 33.395 & 33.421 & 33.497 & 33.629 & 33.886 \\
\hline dimer & $\begin{array}{l}\text { Compression } \\
\text { Benfefitin }\end{array}$ & & 25.65 & 25.65 & 25.65 & 24.92 & 24.49 & 22.42 & 18.51 & 11.97 \\
\hline & PSNR & 31.174 & 28.979 & 28.979 & 28.979 & 28.982 & 28.993 & 29.059 & 29.233 & 29.671 \\
\hline Bark? & $\begin{array}{l}\text { Compression } \\
\text { Benfefin is }\end{array}$ & & 16.89 & 16.89 & 16.89 & 16.87 & 16.69 & 15.72 & 13.83 & 10.01 \\
\hline & PSNR & 31.7773 & 30.029 & 30.029 & 30.029 & 30.03 & 30.043 & 30.113 & 30.622 & 30.662 \\
\hline Glases & $\begin{array}{l}\text { Compression } \\
\text { Benfefin in of }\end{array}$ & & 29.21 & 29.21 & 29.21 & 29.13 & 28.72 & 27.99 & 26.78 & 23.93 \\
\hline & PSNR & 37.248 & 35.542 & 35.542 & 35.542 & 35.541 & 35.542 & 35.551 & 35.595 & 35.844 \\
\hline 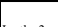 & Compression & & 16.05 & 16.05 & 16.05 & 16.01 & 15.86 & 15.09 & 13.4 & 10.04 \\
\hline Leanther2 & PSNR & 31.422 & 2935 & 2935 & 2935 & 29353 & 29364 & 29426 & 29583 & 29965 \\
\hline & Compression &. & 17.26 & 17.26 & 17.26 & 17.04 & 16.63 & 15.32 & 13.01 & 8.77 \\
\hline Man & $\begin{array}{l}\text { Beneffit in } \\
\text { PSNR }\end{array}$ & 3413 & 31905 & 31905 & 31905 & 31919 & 31937 & 32025 & 32239 & 32821 \\
\hline 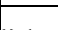 & Compression & . & 20.97 & 20.97 & 20.97 & 20.38 & 19.9 & 17.65 & 13.84 & 8.23 \\
\hline Metalgrates & $\begin{array}{c}\text { Benentif in } \\
\text { PSNR }\end{array}$ & 33.378 & 32.418 & 32.418 & 32.418 & 32421 & 3244 & 32508 & 32628 & 32932 \\
\hline & Compres. & . & 17.59 & 17.59 & 17.59 & 17.54 & 17.25 & 15.93 & 13.42 & 8.94 \\
\hline bubbles2 2 & $\begin{array}{l}\text { Benefif in } \\
\text { PSNR }\end{array}$ & 32.289 & 31.009 & 31.009 & 31.009 & 31.012 & 31.025 & 31.093 & 31.228 & 31.53 \\
\hline & Compression & . & 21.99 & 21.99 & 21.99 & 21.6 & 20.9 & 18.12 & 14.1 & 8.49 \\
\hline Roof2 & $\begin{array}{l}\text { Benefitin } \% \\
\text { PSTR }\end{array}$ & 33.368 & 32.123 & 32.123 & 32.123 & 32.127 & 32.142 & 32.188 & 32,302 & 32719 \\
\hline & Compression & . & 15.56 & 15.56 & 15.56 & 15.53 & 15.29 & 14.23 & 12.24 & 8.63 \\
\hline Sand2 & $\begin{array}{l}\text { Benefitin } \% \\
\text { PSNR }\end{array}$ & 33.19 & 31.947 & 31.947 & 31.947 & 31.949 & 31.962 & 32.019 & 32.145 & 32.395 \\
\hline & Compression & . & 17.73 & 17.73 & 17.73 & 17.61 & 17.45 & 16.82 & 14.18 & 9.15 \\
\hline Sea & $\begin{array}{c}\text { Beneffitin } \% \\
\text { PSNR }\end{array}$ & 38.236 & 37.256 & 37.256 & 37.256 & 37.257 & 37.259 & 37.276 & 37.358 & 37.551 \\
\hline & Compression & 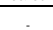 & 11.95 & 11.95 & 11.95 & 11.93 & 11.8 & 11.17 & 9.79 & 7.1 \\
\hline Straw2 & $\begin{array}{l}\text { Benefitin } \% \\
\text { PSNR }\end{array}$ & 32.7811 & 31.592 & 31.592 & 31.592 & 31.593 & 31.604 & 31.664 & 31.792 & 32.069 \\
\hline Wh & Compression & 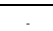 & 18.08 & 18.08 & 18.08 & 18.06 & 17.92 & 17.01 & 15.1 & 11.26 \\
\hline & PSNR & 28.9874 & 26.841 & 26.841 & 26.841 & 26.842 & 26.854 & 26.93 & 27.101 & 27.489 \\
\hline & $\begin{array}{l}\text { Compression } \\
\text { Parsions }\end{array}$ & 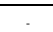 & 20.43 & 20.43 & 20.43 & 20.03 & 19.5 & 17.4 & 13.89 & 8.31 \\
\hline & PSNR & 34.3204 & 33.518 & 33.518 & 33.518 & 33.245 & 33.346 & 33.318 & 33.439 & 33.853 \\
\hline
\end{tabular}




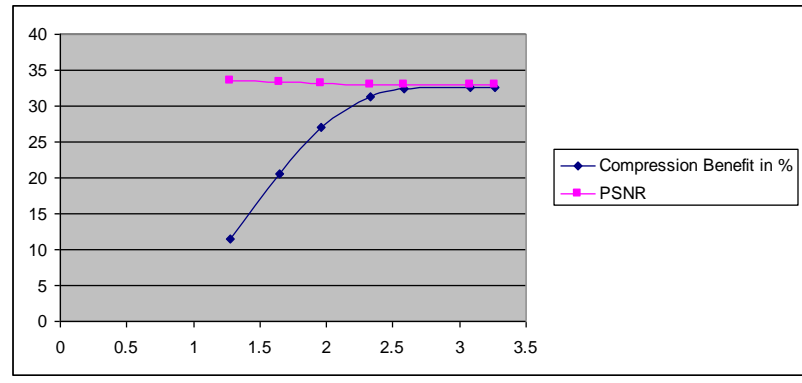

Fig. 5 : Critical Factor Vs Compression Benefit in \% \& Critical Factor Vs PSNR for Image "Gravel" with OutlierMedianDCT

Figure 5 shows As the $\mathrm{C}$ value increases, Compression Benefit increases and PSNR decreases. The variation in PSNR is very small as the $C$ value increases. The PSNR values are very nearer to the PSNR values obtained by conventional JPEG coding.

In a nutshell, the experiments indicated the following,

1. Compression Benefit of algorithms are observed to be MeanDCT $>$ MedianDCT $>$ ConventionalDCT

2. PSNR of algorithms are observed to be MeanDCT $<$ MedianDCT $<$ ConventionalDCT

3. While comparing MeanDCT and the corresponding OutlierDCT, Compression Benefit are observed to be MeanDCT $>$ OutlierMeanDCT (for $\mathrm{C}=1.28$ to 2.58). As the value of $\mathrm{C}$ decreases in the Outlier, Compression Benefit decreases.For $\mathrm{C}=3.08$ to 3.27 Compression Benefit in MeanDCT and OutlierMeanDCT is same.

4. While comparing MeanDCT and the corresponding OutlierDCT, PSNR is observed to be MeanDCT<OutlierMeanDCT. (for $\mathrm{C}=1.28$ to 2.56) As the value of $\mathrm{C}$ decreases in the Outlier, PSNR increases. For $\mathrm{C}=3.08$ to 3.27 PSNR in MeanDCT and OutlierMeanDCT is same.

5. While comparing MedianDCT and the corresponding OutlierDCT, Compression Benefit are observed to be MedianDCT $>$ OutlierMedianDCT (for $\mathrm{C}=1.28$ to 2.58). As the value of $\mathrm{C}$ decreases in the Outlier, Compression Benefit decreases. For $\mathrm{C}=3.08$ to 3.27 Compression Benefit in MedianDCT and OutlierMedianDCT is same.

6. While comparing MedianDCT and the corresponding OutlierDCT, PSNR is observed to be MedianDCT<OutlierMedianDCT (for $\mathrm{C}=1.28$ to 2.56 ) as the value of $\mathrm{C}$ decreases in the Outlier, PSNR increases. For $\mathrm{C}=3.08$ to 3.27 PSNR in MedianDCT and OutlierMedianDCT is same.

7. All the retrieved images based on Conventional JPEG system and Mean, Median \& Outlier based JPEG system are almost same for visual appearance.

8. All the error images based on Conventional JPEG system and Mean ,Median \& Outlier based JPEG system are negligible

In the recent years, value added multi-media services are gaining importance. Here, the consumer will be billed in accordance with the quality of service he has enjoyed. All of the algorithms are best suitable at this junction as they have freedom to control the quality with decreasing $\mathrm{C}$.

\section{CONCLUSIONS}

In this paper, new MeanDCT, MedianDCT, OutlierMeanDCT \& OutlierMedianDCT based JPEG compression algorithms are proposed. The authors have compared these MeanDCT, MedianDCT, OutlierMeanDCT \& OutlierMedianDCT based JPEG compression algorithms with Conventional JPEG compression. From these experiments it is evident that these approaches gives better compression ratios compared to conventional JPEG. The PSNR resulting from the approach is slightly less than Conventional approach. The PSNR resulting from OutlierMedianDCT with lowest critical factor is almost same as conventional approach. Highest Compression Benefit is achieved from OutlierMeanDCT with highest critical factor. However, all the decoded images resulting from this approach and original images are almost the same in human perception point of view.

\section{REFERENCES}

[1] Muhammad F.Sabir, Hamid Rahim Sheikh, Robert W.Heath, Alan C.Bovik" A Joint Source-Channel Distortion Model for JPEG Compressed Images" IEEE Transictions on Image Processing Vol 15, No 6, pp1349. 1364 June 2006

[2] Cung Nguyen "Detecting Computer -Induced Errors in Remote-Sensing JPEG Compression Algorithms" IEEE Transictions on Im age Processing Vol 15, No 7, pp17281739 July 2006

[3] R.C.Gonzalez and R.E.Woods "Digital Image Processing", 2nd Edition Addison Wesley, USA ISBN 0-201-60078, 1993sz

[4] The USC-SIPI image database (http://sipi.usc.edu/database). Signal and image processing institute Ming Hgieh Department of Electrical Engineering.

[5] Andrew B.Watson "Image Compression using the discrete cosine transform" Mathematica Journal 4 (1), 1994, p-81-88

[6] Ken cabeen and peter gent," Image compression and the discrete cosine transform", Math 45 college of redwoods

[7] K. Kantapanit and W. Wiriyasuttiwong. "Face Recognition by Edge Detection of JPEG Compressed Images and Backpropagation Neural Network" The Engineering Journal of Siam University. Volume 3, Year 2 , July-December, 2000.

[8] "Mean filtering, Smoothing, Averaging, Box filtering",http://homepages.inf.ed.ac.uk/rbf/HIPR2/mean. htm

[9] "Median filtering, Rank filtering" http://homepages.inf. ed.ac.uk/rbf /HIPR2/median.htm

[10] "Cofidence Intervals",http://www.stat.yale.edu/ courses/1997-98/101/confint.htm

[11] "Working with outliers", http://en.wikipedia.org/ wiki/ outlier

[12] "JPEG Artifacts “,http://graphicssoft.about.com/od/ glossary/g/jpegartifacts.htm

[13] "JPEG Artifacts", http://www.scantips.com/ basics9jb.html 